\title{
Investigation of Dermis-derived Hydrogels for Wound Healing Applications
}

\author{
Holger Engel ${ }^{1,2, *}$, Shu-Wei Kao ${ }^{1,3, *}$, Jeffery Larson ${ }^{4,5}$, Shiri Uriel ${ }^{4}$, Bin Jiang ${ }^{4,5}$, Eric M. Brey ${ }^{4,5}$, \\ Ming-Huei Cheng ${ }^{1,3}$
}

Background: Wound healing and skin tissue engineering are mediated, in part, by interactions between cells and the extracellular matrix (ECM). A subset of the ECM, basement membranes $(\mathrm{BM})$, plays a vital role in regulating proper skin healing and function.

Methods: ECM-rich, tissue-specific hydrogels were extracted and assembled from dermis samples. These hydrogels contain BM proteins vital to skin regeneration, including laminin $\beta 3$, collagen IV, and collagen VII. The extracts could be assembled to form hydrogels by either temperature or $\mathrm{pH}$ mechanism, with the mechanical properties and structure varying with the mechanism of assembly. A wound healing model was developed to investigate the ability of these hydrogels to enhance healing with a single application in vivo.

Results: The $\mathrm{pH}$, but not temperature gels were easily applied to the wounds. There were no signs of increased inflammation due to the application of the hydrogels. The width of granulation tissue at the first week was reduced $(p=0.064)$ relative to controls with the application of hydrogel. There

\section{At a Glance Commentary}

Scientific background of the subject

Poor wound healing remains a significant clinical challenge. Biomaterials that mimic the extracellular matrix (ECM) structure of tissues may enhance healing. ECM-rich biomaterials were isolated from dermis and investigated in a rodent wound healing model.

\section{What this study adds to the field}

The hydrogels could be easily applied to the wounds, did not increase inflammation, and reduced granulation tissue area within 1 week. Dermis-derived hydrogels have promise for skin regeneration, but their poor mechanical strength and rapid degradation may hinder their biological effects

Conclusions: Dermis-derived hydrogels contain BM proteins important for skin regeneration. They can be easily applied, but their poor mechanical strength and rapid degradation may hinder their biological effects. (Biomed J 2015;38:58-64)

Key words: basement membrane, dermis, extracellular matrix, hydrogel, skin, wound healing

$\mathrm{C}$ Yell interactions with the extracellular matrices (ECM) in general and specifically with the dermal basement membrane (BM) play an important role in normal skin physiology and wound healing. ${ }^{[1]}$ The BM is a sheet of fibrous extracellular material upon which the basal surfaces of epithelial cells rest. The degradation of existing matrix and synthesis of new BM is a limiting step in wound closure. ${ }^{[2]}$ For this reason, ECM-based natural and synthetic materials have been investigated for applications in the regeneration or reconstruction of skin. ${ }^{[3,4]}$ However, the materials investigated often feature ECM properties derived from areas other than skin. Materials that contain ECM features

\footnotetext{
*These authors contributed equally to the work.

From the ${ }^{1}$ Department of Plastic and Reconstructive Surgery, Chang Gung Memorial Hospital at Linkou, Chang Gung University College of Medicine, Taoyuan, Taiwan; ${ }^{2}$ Department of Plastic, Reconstructive, Aesthetic and Hand Surgery, Medical Center Kassel, Kassel, Germany; ${ }^{3}$ Center for Tissue Engineering, Chang Gung Memorial Hospital at Linkou, Chang Gung University College of Medicine, Taoyuan, Taiwan; ${ }^{4}$ Department of Biomedical Science and Engineering, Pritzker Institute, Illinois Institute of Technology, Chicago, IL, USA; ${ }^{5}$ Research Service, Edward Hines, Jr. V. A. Hospital, Hines, IL, USA.

Received: Jun. 13, 2013; Accepted: Jan. 16, 2014

Correspondence to: Prof. Ming-Huei Cheng, Department of Plastic and Reconstructive Surgery, Chang Gung Memorial Hospital at Linkou. 5, Fusing St., Gueishan, Taoyuan 333, Taiwan (ROC). Tel: 886-3-3281200 ext. 3355; Fax: 886-3-3970370;

E-mail: minghueicheng@gmail.com
}

DOI: $10.4103 / 2319-4170.132899$ 
specific to skin may enhance tissue regeneration. We have recently developed a novel method for generating ECM-rich, tissue-derived hydrogels with chemical and physical properties that vary depending on the tissue source. ${ }^{[5,6]}$ Hydrogels developed from this technique have shown promise in tissue engineering applications due to their ability to stimulate vessel assembly in vitro and vascularized tissue formation in vivo. ${ }^{[5-7]}$ Hydrogels assembled from dermal tissue contain BM proteins, including laminin $\beta 3$, collagen IV, and collagen VII, which are essential for proper skin function. ${ }^{[5,6]}$ Dermis-based hydrogels may provide benefit for applications in wound healing or tissue engineering of skin.

The ability to evaluate biomaterials for wound healing applications requires a quantifiable and consistent animal model in which wound healing occurs via granulation tissue formation, angiogenesis, and re-epithelialization. Avoiding healing by wound contraction, which can account for as much as $90 \%$ of wound closure, is crucial in rodents which are "loose-skinned" animals. ${ }^{[8,9]}$ In order to focus on the important components of wound healing, it is also essential to provide a stable wound environment for reliable data evaluation and analysis. In this study, we examined the physical and mechanical properties of dermis-derived hydrogels and evaluated their application in a rodent wound healing model that heals with minimal wound contraction.

\section{METHODS}

\section{Extraction}

All the animal protocols received approval from the Institutional Animal Care and Use Committee of our institution. Full-thickness skin was harvested from Sprague Dawley rats (250-350 g) immediately following euthanasia by intracardial injection of potassium chloride. Subcutaneous fat and epidermis were carefully dissected away from the dermis. ECM-rich extracts were isolated from the dermis samples for gelation, as described previously. ${ }^{[5,6]}$ Briefly, the dermis samples were cut into small sections (1-2 mm in thickness) and suspended in dispase solution (Sigma, St. Louis, MO, USA; $2 \mathrm{ml} / \mathrm{g}$ of tissue) at $4^{\circ} \mathrm{C}$ for $15 \mathrm{~min}$. The tissues were then rubbed over a cell sieve before homogenization in a high salt buffer containing protease inhibitors [0.05 M Tris $\mathrm{pH}$ 7.4, 3.4 M sodium chloride $(\mathrm{NaCl}), 4 \mathrm{mM}$ of ethylenediaminetetraacetic acid, $2 \mathrm{mM}$ of $\mathrm{N}$-ethylmaleimide, $0.001 \mathrm{mg} / \mathrm{ml}$ pepstatin, $0.01 \mathrm{mg} / \mathrm{ml}$ aprotonin, $0.001 \mathrm{mg} / \mathrm{ml}$ leupeptin, $2 \mathrm{mM}$ sodium orthovanadate, $1 \mathrm{mM}$ phenylmethylsulfonyl fluoride (Sigma)]. The tissue was then incubated in $2 \mathrm{M}$ urea buffer $(0.15 \mathrm{M}$ sodium chloride, 0.05 Tris $\mathrm{pH} 7.4 ; 1 \mathrm{ml} / \mathrm{g}$ of tissue) overnight at $4^{\circ} \mathrm{C}$. The mixture was centrifuged at $14,000 \mathrm{~g}$ for $20 \mathrm{~min}$ and the supernatant containing the extracted ECM proteins was stored at $4{ }^{\circ} \mathrm{C}$ until further use.

\section{Characterization}

Dermal extracts were induced to assemble into gels by two processes: (1) incubation at $37^{\circ} \mathrm{C}$ and (2) reduction of $\mathrm{pH}$ to $\sim 4$. 0 by the addition of acetic acid. The structure of the materials was characterized using atomic force microscopy (AFM). The samples were imaged using a multimode Nanoscope IIIa AFM (Digital Instruments, Santa Barbara, CA, USA). Imaging was performed with a silicon $\mathrm{Si}_{3} \mathrm{~N}_{4}$ cantilever tip at a frequency of 20-30 kHz. The Picoscan 3000 program (Agilent Technologies Molecular Imaging, Santa Clara, CA, USA) allowed viewing and analysis of the resulting AFM images. The mean distance between elevations was calculated and estimated as the pore diameter. Elevations were determined based on the altitude measured by the cantilever, and the distance between two close elevation peaks was calculated by the Picoscan program. A minimum of 10 measurements per view was taken.

AFM was also used to measure material stiffness based on force-displacement data. The procedure used has been described previously. ${ }^{[10]}$ The sample was moved in the $z$ (vertical) direction through a known displacement, while monitoring the deflection of the AFM cantilever. The measured cantilever deflection, $\Delta d$, depends on both the displacement of the tip and deformation of the sample: $d-d_{0}=z-z_{0}-\delta$, where $z_{0}, d_{0}$, and $\delta$ are the contact point, the free deflection value, and the deformation, respectively. As the loading force is due to the deflection of the cantilever spring, it has a magnitude of $k\left(d-d_{0}\right)$, where $k$ is the spring constant known to be $0.3 \mathrm{~N} / \mathrm{m}$ when the cantilever is modeled as a rigid cone connected to a spring. The Young's Modulus $(E)$ of the sample can then be calculated using Equation 1 with constants: $k=0.3 \mathrm{~N} / \mathrm{m}, v=0.5, \alpha=35^{\circ}$, as well as the $\Delta z$ and $\Delta d$ values read from the force spectra: ${ }^{[10]}$

$z-z_{0}=d-d_{0}+\frac{k \pi\left(1-v^{2}\right)\left(d-d_{0}\right)}{2 E \tan \alpha}$

Using AFM contact mode in liquid $\left(\mathrm{dH}_{2} \mathrm{O}\right)$, the sample mechanical properties were measured based on a minimum of four generated force displacement curves per gel assembly condition ( $\mathrm{pH}$ and thermal).

Volume fractions were also measured for gels formed by both acid and temperature mechanisms of gelation. The extracts were gelled (four per group) and swelled for $24 \mathrm{~h}$ in Tris-buffered saline (TBS). The next day, TBS was removed and the swelled weight $\left(W_{\text {wet }}\right)$ determined. The gels were then placed in a non-humidified incubator at $37^{\circ} \mathrm{C}$ for 24 $\mathrm{h}$ and weighed again $\left(W_{\text {dry }}\right)$ to determine the swelling ratio as follows: ${ }^{[11]}$

$S R=\frac{W_{w e t}}{W_{d r y}}$

The density of the ECM proteins can be estimated as $1.35 \mathrm{~g} / \mathrm{cm}^{3}$ based on theoretical calculations. ${ }^{[12]}$ Therefore, 
the volume fractions of the hydrogels were calculated using protein and water densities of 1.35 and $1.0 \mathrm{~g} / \mathrm{cm}^{3}$, respectively. Volume fraction $(\phi)$ was calculated as follows:

$$
\phi=\frac{W_{d r y} * \frac{1}{1.35 g / \mathrm{cm}^{3}}}{\left[W_{d r y} * \frac{1}{1.35 g / \mathrm{cm}^{3}}+\left(W_{w e t}-W_{d r y}\right) * \frac{1}{1.0 \mathrm{~g} / \mathrm{cm}^{3}}\right]}
$$

\section{Wound healing model}

A modified excisional full-thickness wound healing model was developed in order to evaluate the dermis hydrogels in a wound healing setting. Sprague Dawley rats $(250-350 \mathrm{~g})$ were anesthetized with isoflurane, shaved, and prepared according to standard sterile procedure. For a consistent wound location, a midline was drawn on the back of the rat. The midpoint between the ears was marked, and $3 \mathrm{~cm}$ caudally, two circles of $22 \mathrm{~mm}$ diameter were drawn on their back. A full-thickness excisional wound was made in the center of each circle with an 8-mm punch. A semi-rigid (external/internal diameter 22/12 mm) silicone ring custom-made out of a standard silicon sheet (Perthese ${ }^{\circledR}$; Perouse Plastie Inc., Bornel, France) was centered over the wound and affixed using cyanoacrylate glue. The outer side and middle part of the ring were sutured alternately with 4-0 Ethilon ${ }^{\circledR}$ to guarantee proper ring fixation on the back of the rat. The skin edges of the wound inside the silicone ring $(2 \mathrm{~mm})$ were stitched and fixed to the inner circle of the ring to minimize wound contracture.

Dermis hydrogels were prepared within $24 \mathrm{~h}$ of application to the wound model. Hydrogel extracts were dialyzed against media containing gentamicin for sterilization and stored at $4^{\circ} \mathrm{C}$ prior to gelation and application to the wounds. The hydrogels maintained their structure after gelation and were easily applied to the wound using a spatula. After application of dermis-derived hydrogels (wounds without gels were used in control cases), the wound was closed with a self-adhesive sheet $\left(\right.$ Tegaderm $\left.^{\circledR}\right)$ creating a stable wound. A rat collar (Braintree Scientific, Inc., Braintree, MA, USA) was used to prevent removal of the wound chambers. At 1 , 2 , and 3 weeks, the wound chambers were removed and square full-thickness skin samples (including the wound region and surrounding tissue) were harvested and prepared for histological and immunohistochemical characterization.

\section{Evaluation}

Samples were formalin-fixed, paraffin-embedded, and sectioned at $5 \mu \mathrm{m}$ thickness. The samples were oriented such that the sections passed through the center of the wounds. Sections were stained with hematoxylin and eosin (H and E) and Masson's trichrome. Immunohistochemical staining for CD31 was performed as described previously. ${ }^{[6]}$ Briefly, paraffin-embedded sections were deparaffinized and rehydrated prior to antigen retrieval using Target Retrieval Solution (Dako, Carpinteria, CA, USA). Specimens were then treated with $3 \% \mathrm{H}_{2} \mathrm{O}_{2}$ to block endogenous peroxidase activity and with $5 \%$ goat serum to prevent nonspecific binding. Rabbit anti-CD31 (Santa Cruz Biotechnology, Santa Cruz, CA, USA) was applied to the sections overnight at $4^{\circ} \mathrm{C}$, followed by identification with a biotinylated anti-rabbit secondary antibody using a Vectastain Elite ABC kit (Vector Labs, Burlingame, CA, USA). Counterstaining was performed with hematoxylin.

Stained sections were digitally imaged using an Axiovert 200 inverted microscope with $5 \times$ objective $(1.25 \mu \mathrm{m} / \mathrm{pixel})$ for histological analysis. Histological parameters, including the length of re-epithelialization, the width of the unclosed wound, and the width of granulation tissue, were quantified as described previously using Axiovision AC (Carl Zeiss, Oberkochen, Germany) [Figure 1]. ${ }^{[13]}$ The length of re-epithelialization is defined as the length of neoepidermis along the surface of newly regenerated granulation tissue. The length of unclosed wound is defined as the distance between the two ends of the newly regenerated epidermis. The width of granulation tissue is defined as the narrowest distance between the two ends of granulation tissue. Two independent sections were measured per wound.

Blood vessel density was analyzed by counting the total number of CD31-positive vessels in each view area $(20 \times$ objective, $0.31 \mu \mathrm{m} /$ pixel, $485 \mu \mathrm{m} \times 328 \mu \mathrm{m})$ and quantified as vessels/area. For each tissue section, three images including two from the granulating tissue edges and one from the center of the wound were evaluated. ${ }^{[14]}$

Inflammatory response was also evaluated for the $\mathrm{H}$ and E-stained sections at 1 week. The total number of inflammatory cells was counted manually in each view area (40 $\times$ objective, $0.16 \mu \mathrm{m} /$ pixel, $218 \mu \mathrm{m} \times 164 \mu \mathrm{m})$ and the density of cells per area quantified. Two images were taken per section and two independent sections were measured per wound. ${ }^{[15]}$

\section{Statistical analysis}

Data on hydrogel properties were compared using analysis of variance (ANOVA) with a Tukey-Kramer post test. Paired Student's $t$ tests were used for comparison

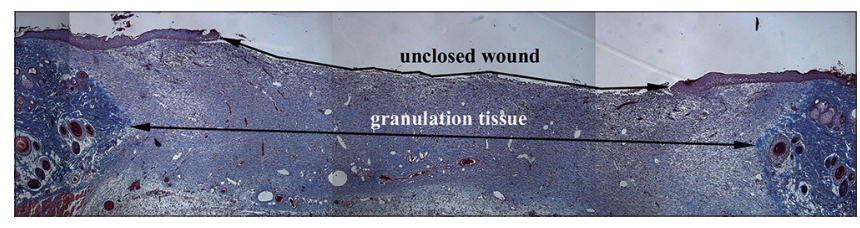

Figure 1: Sample stained with Masson's trichrome showing the features measured to determine widths of the unclosed wound and granulation tissue. 
of histomorphometric data. For all comparisons, $p<0.05$ was considered statistically significant.

\section{RESULTS}

\section{Hydrogel properties}

Extracts from dermis contain a complex mixture of proteins and polysaccharides [Table 1]. ${ }^{[5,6]} \mathrm{BM}$ proteins important for proper dermal function, including collagen VII, collagen IV, laminin $\beta 3$, nidogen, and fibronectin, are present in the extracts. ${ }^{[5,6]}$ The extracts can be induced to rapidly assemble into gels by $\mathrm{pH}$ or temperature mechanism. The temperature mechanism requires hours for complete gelation $(168.6 \pm 52.0 \mathrm{~min})$, while exposure to acidic conditions results in assembly in less than $1 \mathrm{~min}(0.5 \pm 0.2 \mathrm{~min}){ }^{[6]}$ Gels formed in both approaches result in a fibrous network similar in structure to $\mathrm{BM}$ in vivo. While the composition of the gels is the same whether gelled by $\mathrm{pH}$ or thermal mechanism, the resultant gels have differences in physical and mechanical properties. Both conditions resulted in gels with high water content as indicated by swelling ratios greater than 45 and protein volume fractions less than 0.02 [Table 2]. Gels formed by $\mathrm{pH}$ mechanism had slightly less water content than temperature gels, but the differences were not statistically significant.

AFM was used to further examine the gel properties.

Table 1: Composition of dermis derived hydrogels

\begin{tabular}{lc}
\hline Analysis & Result \\
\hline Protein concentration $(\mathrm{mg} / \mathrm{ml})$ & $10-17$ \\
Collagen $(\%)$ & $59.0 \pm 5.6$ \\
Sulfated glycosaminoglycans $(\%)$ & $0.40 \pm 0.08$ \\
Glycoprotein $(\%)$ & $26.8 \pm 0.4$ \\
BM proteins & Collagen VII \\
& Collagen IV \\
& Laminin $\beta 3$ \\
& Nidogen \\
Growth factors (ng/ml) & Fibronectin \\
& FGF-1: $11.0 \pm 4.0$ \\
\hline Data for this table comes from Uriel et al. 2009 and Cheng et al. 2010
\end{tabular}

Data for this table comes from Uriel et al., 2009 and Cheng et al., 2010

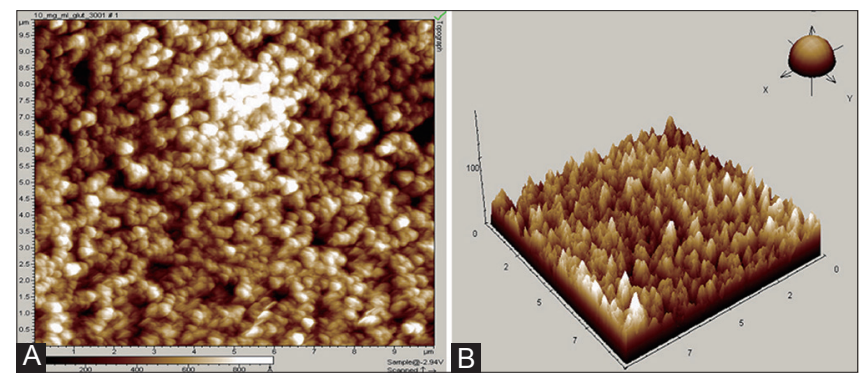

Figure 2: The pore structure of dermis gels was evaluated using atomic force microscopy. (A) Three-dimensional and (B) two-dimensional reconstructions of the porous surface of the gels.
The mean pore diameter determined from the AFM images [Figure 2] under hydrated conditions was $384 \pm 160 \mathrm{~nm}$, which is similar to the mean pore diameter calculated from scanning electron microscopy (SEM) images [Table 2]. Gels induced by a $\mathrm{pH}$ change were difficult to image due to adhesion of the tip to the gel surface. Therefore, the surface images were blurry which hindered determination of pore size. No statistical differences $(p>0.05)$ were found between the pore structure measurements calculated using SEM and AFM. The stiffness of the gels varied depending on the mechanism of gelation. Temperature gels were softer than $\mathrm{pH}$-induced gels (Young's modulus $1.2 \pm 0.8 \mathrm{~Pa}$ vs. $94.9 \pm 24.1 \mathrm{~Pa}$ for temperature and $\mathrm{pH}$, respectively; $p<0.05$ ).

\section{Wound healing model}

Gels were formed by both temperature and $\mathrm{pH}$ mechanisms. The temperature gels were too fragile to be transferred to the wound. The gels formed by $\mathrm{pH}$ mechanism could be easily handled [Figure $3 \mathrm{~A}]$ and directly applied to the surface of the wound bed using a spatula [Figure 3B]. Affixation of the silicone ring to the skin using cyanoacrylate glue was safe and fast. The animals appeared to tolerate the wounds and the silicone rings very well. Macroscopically, the wounds appeared to follow the wound healing sequence of granulation and re-epithelialization without evidence of wound contraction.

\section{Histological evaluation}

Samples were harvested at 1, 2, and 3 weeks for his-

Table 2: Properties of dermal gels formed by $\mathrm{pH}$ and temperature mechanisms

\begin{tabular}{lcc}
\hline & $\mathrm{pH}$ & Temperature \\
\hline Gelation time $(\mathrm{min})^{\dagger}$ & $0.5 \pm 0.2^{*}$ & $168.6 \pm 52.0$ \\
Fiber size $(\mathrm{nm})^{\dagger}$ & $32 \pm 15^{*}$ & $55 \pm 14$ \\
Pore Size $(\mathrm{nm})^{\dagger}$ & $160 \pm 64^{*}$ & $359 \pm 143$ \\
Young's modulus (Pa) & $94.9 \pm 24.1^{*}$ & $1.2 \pm 0.8$ \\
Volume fraction (unitless) & $0.017 \pm 0.002$ & $0.015 \pm 0.003$ \\
Swelling ratio (v/v) & $46.2 \pm 6.5$ & $48.2 \pm 12.7$ \\
\hline *indicates significant differences between the two hydrogels $(p<0.05)$. \\
'Data are from Uriel et al. 2009
\end{tabular}

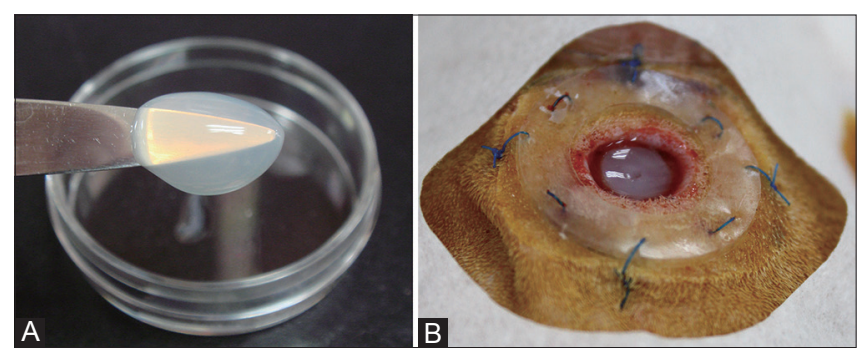

Figure 3: Images of dermis-derived hydrogel formed by the $\mathrm{pH}$ mechanism. (A) Dermis-derived hydrogel prior to application is easily handled and (B) applied to the full-thickness excisional wound. Note the silicone ring present to inhibit wound contraction. 
tological evaluation. The gels had completely degraded by 1 week as there were no signs of the gels either visually or histologically. The density of inflammatory cells was the same in both gel-treated and control wounds [Figure 4]. At 1 week [Figure 5A], both gel-treated and control wounds consisted primarily of granulation tissue, with an epidermis forming along the surface from the wound edges. In most wounds, the epithelium was absent in a region in the center of the wound surfaces. Two and three weeks after the surgery [Figure 5B and C], most wounds were covered with an intact layer of epidermis, and granulation tissue was replaced by mature collagen from the wound edges. The hydrogel-treated groups had a smaller average width of granulation tissue when compared to untreated wounds at 1 week using paired statistical analysis [Figure 6C] $(p=0.064)$. However, there were no statistical differences in the length of re-epithelialization or the length of unclosed wound between hydrogel-treated groups and controls when applying the same analysis [Figure 6A and B]. By 2 and 3 weeks, the values were the same for both treated and untreated wounds. At 1 week, only one out of five of the wounds in both control and gel-treated groups were closed. At 2 and 3 weeks, four out of five of the wounds in gel-treated groups had closed and all the control wounds had closed.

Dermis-derived hydrogels have been shown to support vessel assembly in vitro and vascularized tissue formation in vivo. ${ }^{[5,6]}$ Immunohistochemical staining of wound tissue sections for CD31, an endothelial cell marker, was used to evaluate vessel density in the wound bed. Robust vascularization was seen in all groups. No significant difference in blood vessel density was found when comparing control and the dermis hydrogel-treated groups [Figure 6D].

\section{DISCUSSION}

The ECM plays a very important role in skin physiology and wound healing. While the 3D fibrous network structure of dermis-derived hydrogels is similar to the in vivo nanostructure of BM, the stiffness is much lower than native skin. ${ }^{[5]}$ It is difficult to measure mechanical properties of the

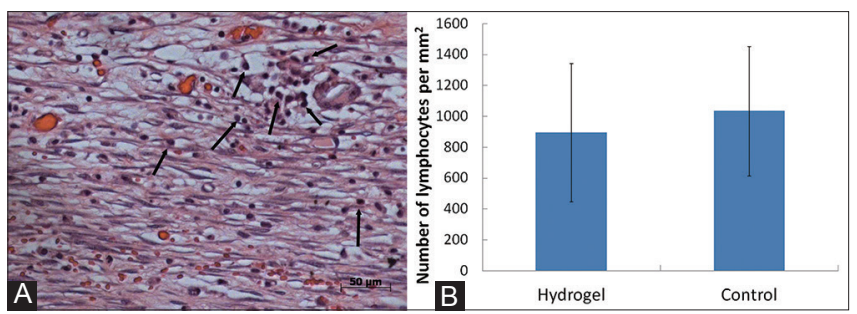

Figure 4: Evaluation of inflammation in wounds. (A) $\mathrm{H}$ and $\mathrm{E}$ staining of wound granulation tissue 1 week post surgery in a wound treated with a dermis-derived hydrogel. The black arrows indicate lymphocytes. Scale bar $=100 \mu \mathrm{m}$. (B) The density of the lymphocytes in the granulation tissue during wound healing $(n=5)$. Values are shown as means $\pm \mathrm{SD}$.
BM directly, but the Young's modulus of intact human skin has been recorded between $4.2 \times 10^{5}$ and $8.5 \times 10^{5} \mathrm{~Pa}^{\left[{ }^{[16]}\right.}$ The values are much higher than those of dermis-derived hydrogels formed by either temperature $(1.2 \pm 0.8 \mathrm{~Pa})$ or $\mathrm{pH}$ mechanism $(94.9 \pm 24.1 \mathrm{~Pa})$. The lower stiffness may result from the absence of cells or the lack of covalent crosslinking of the materials. The methods used for extraction and gel assembly result in proteins that are held together by fairly weak secondary bonds. A variety of methods are available for increased crosslinking of ECM-based materials that may be used to increase the stiffness of the tissue-derived materials. ${ }^{[17]}$

The poor mechanical properties of the temperature gels made them difficult to handle. However, $\mathrm{pH}$ gels could be easily handled and applied to the wounds. The quantification of lymphocyte density showed no significant difference between the groups treated with or without hydrogels for the first week tissues, suggesting that the hydrogels did not cause a prolonged or increased inflammatory response. While wounds treated with dermis-derived hydrogel exhibited a smaller width of granulation tissue at 1 week relative to controls, the wound healing improvements were not significant. This is somewhat surprising as dermis-derived hydrogels not only contain multiple BM proteins and glycoproteins that are essential for wound healing, but also contain a variety of growth factors [Table 1]. One potential reason was that the hydrogels suffer from rapid degradation and clearance in vivo. It is possible that a single application of the hydrogels at the very beginning of wound healing does not provide lasting effects.

Rodents have a long history of being employed as wound healing models because of their wide availability and suitable size. ${ }^{[18]}$ However, a major difference between rodent and human wound healing mechanisms is that rodents

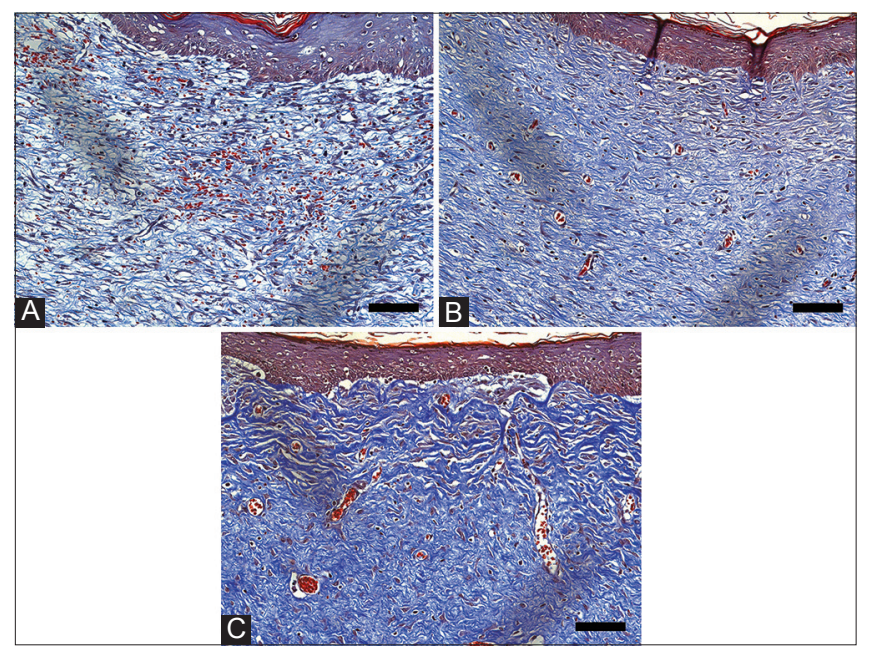

Figure 5: Masson's trichrome staining of wound tissue sections for (A) 1 week, (B) 2 weeks, and (C) 3 weeks after surgery. Scale bar $=50 \mu \mathrm{m}$. Trichome stains collagenous fibrous connective tissue blue, which thickened and matured over time. 


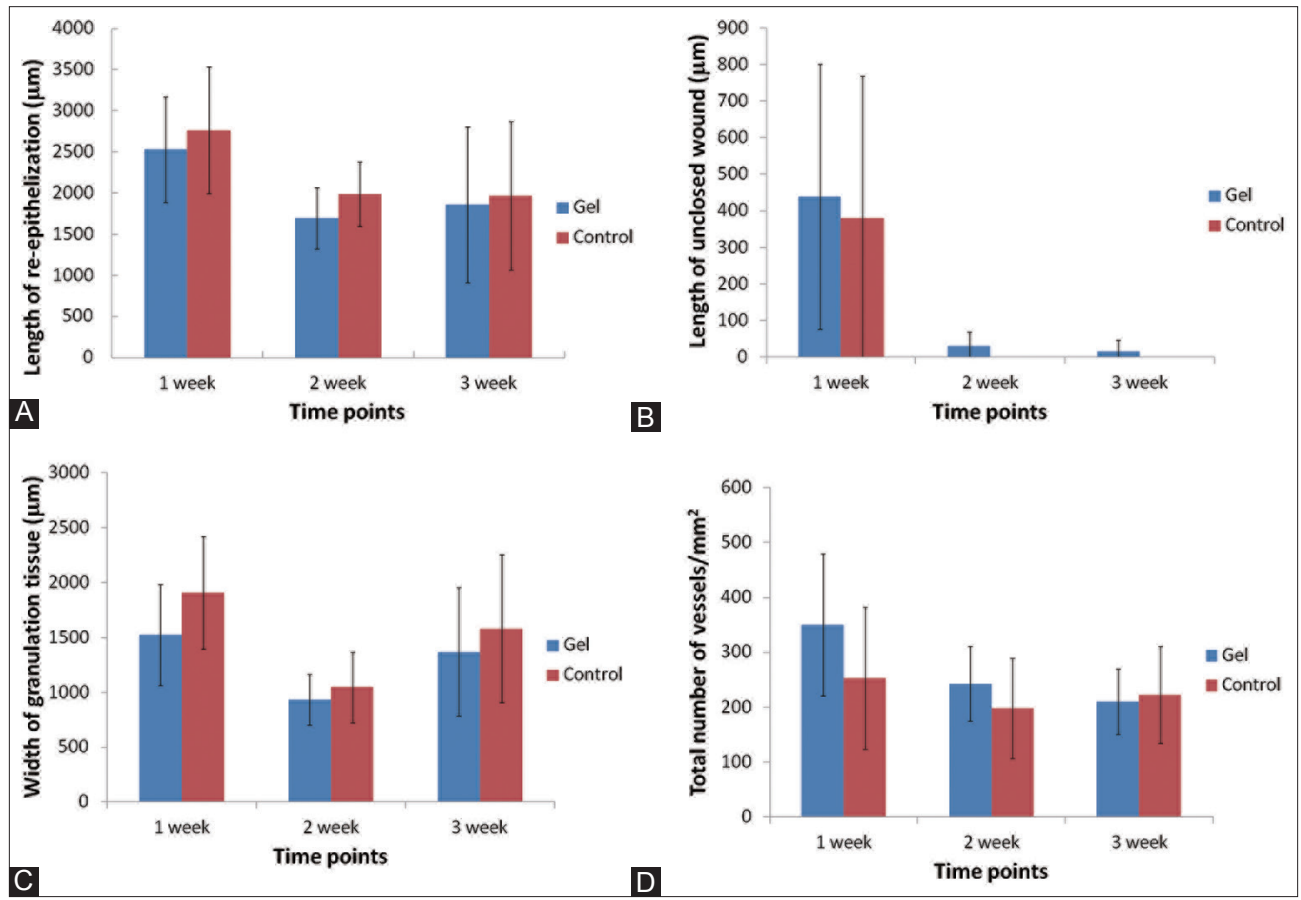

Figure 6: Histological and immunohistochemical analysis of wound healing model $(n=5)$ treated with and without dermis-derived hydrogel for 1, 2, and 3 weeks. Effect of dermis-derived hydrogel on (A) the length of re-epithelialization, (B) the length of unclosed wound, (C) the width of granulation tissue, and (D) vessel density. Values are shown as means \pm SD.

have a mobile integument and are called "loose-skinned" animals. In rodents, contraction accounts for almost the entire wound closure process, while in humans, contraction accounts for little more than a third of the closure process. ${ }^{[19]}$ In our wound healing animal model, we fixed a silicone splint ring to each full-thickness excisional wound to avoid healing by means of contraction. Splinting the wound allowed us to more closely mimic the wound healing response in human dermis through generation of granulation tissue and re-epithelialization with little wound contraction. This model is easily reproducible and should provide a valuable tool for evaluation of new materials for wound healing and tissue engineering applications.

The poor mechanical properties and rapid degradation of the hydrogels may have hindered their success in improving wound healing despite the presence of essential $\mathrm{BM}$ proteins and growth factors. Several modifications may allow improving these properties. First, the dermis-derived hydrogels could be covalently crosslinked. The crosslinking could improve the mechanical properties and hinder degradation. In addition, multiple applications of the hydrogels to the healing wound may accelerate regeneration as has been shown with other ECM-based materials used clinically. ${ }^{[20]}$

\section{Conclusion}

A technique is described for the development of biomaterials rich in tissue-specific ECM. These materials provide environments that may be well suited for wound healing mechanisms due to their tissue-like compositions and architecture. The gelation and mechanical and physical properties of the gels are described, and the hydrogels are evaluated in a murine wound healing model that minimizes contraction. The hydrogels did not exhibit an increased inflammatory response in this model and exhibited a trend toward increased resolution of the granulation tissue formed. While these materials may have potential for wound healing, their poor mechanical properties and rapid degradation likely reduced their effectiveness in this model. Further modifications and evaluation of these dermis-derived hydrogels are being made to optimize their effectiveness toward the treatment of full-thickness wounds.

\section{Acknowledgments}

The authors would like to acknowledge the assistance of Dr. Rong Wang with the AFM studies. This work was supported by funding from Taiwan Ministry of Science and Technology (Grant NSC 99-2314-B-182-015-MY3), the Veterans Administration, and the National Science Foundation (DIIS 1125412).

\section{REFERENCES}

1. Baranowsky A, Mokkapati S, Bechtel M, Krugel J, Miosge N, Wickenhauser $\mathrm{C}$, et al. Impaired wound healing in mice lacking the basement membrane protein nidogen 1. Matrix Biol 2010;29:15-21.

2. Singer AJ, Clark RA. Cutaneous wound healing. N Engl J Med 1999;341:738-46. 
3. Yeh JT, Lin CH, Lin YT. Skin grafting as a salvage procedure in diabetic foot reconstruction to avoid major limb amputation. Chang Gung Med J 2010;33:389-96.

4. Yang JY, Chuang SS, Yang WG, Tsay PK. Egg membrane as a new biological dressing in split-thickness skin graft donor sites: A preliminary clinical evaluation. Chang Gung Med J 2003;26:153-9.

5. Uriel S, Labay E, Francis-Sedlak M, Moya ML, Weichselbaum RR, Ervin N, et al. Extraction and assembly of tissue-derived gels for cell culture and tissue engineering. Tissue Eng Part C Methods $2009 ; 15: 309-21$.

6. Cheng MH, Uriel S, Moya ML, Francis-Sedlak M, Wang R, Huang JJ, et al. Dermis-derived hydrogels support adipogenesis in vivo. J Biomed Mater Res A 2010;92:852-8.

7. Uriel S, Huang JJ, Moya ML, Francis ME, Wang R, Chang SY, et al. The role of adipose protein derived hydrogels in adipogenesis. Biomaterials 2008;29:3712-9.

8. Hayward PG, Robson MC. Animal models of wound contraction. Prog Clin Biol Res 1991;365:301-12.

9. Galiano RD, Michaels J $5^{\text {th }}$, Dobryansky M, Levine JP, Gurtner GC. Quantitative and reproducible murine model of excisional wound healing. Wound Repair Regen 2004;12:485-92.

10. Jacot JG, Dianis S, Schnall J, Wong JY. A simple microindentation technique for mapping the microscale compliance of soft hydrated materials and tissues. J Biomed Mater Res A 2006;79:485-94.

11. Urech L, Bittermann AG, Hubbell JA, Hall H. Mechanical properties, proteolytic degradability and biological modifications affect angiogenic process extension into native and modified fibrin matrices in vitro. Biomaterials 2005;26:1369-79.
12. Quillin ML, Matthews BW. Accurate calculation of the density of proteins. Acta Crystallogr D Biol Crystallogr 2000;56:791-4.

13. van Rossum M, Vooijs DP, Walboomers XF, Hoekstra MJ, Spauwen PH, Jansen JA. The influence of a PHI-5-loaded silicone membrane, on cutaneous wound healing in vivo. J Mater Sci Mater Med 2007;18:1449-56.

14. Pietramaggiori G, Scherer SS, Cervi D, Klement G, Orgill DP. Tumors stimulate platelet delivery of angiogenic factors in vivo: An unexpected benefit. Am J Pathol 2008;173:1609-16.

15. Bhardwaj U, Sura R, Papadimitrakopoulos F, Burgess DJ. Controlling acute inflammation with fast releasing dexamethasone-PLGA microsphere/pva hydrogel composites for implantable devices. J Diabetes Sci Technol 2007;1:8-17.

16. Agache PG, Monneur C, Leveque JL, De Rigal J. Mechanical properties and Young >s modulus of human skin in vivo. Arch Dermatol Res 1980;269:221-32.

17. Pilipchuk SP, Vaicik MK, Larson JC, Gazyakan E, Cheng MH, Brey EM. Influence of crosslinking on the stiffness and degradation of dermis-derived hydrogels. J Biomed Mater Res A 2013;101:2883-95.

18. Dorsett-Martin WA. Rat models of skin wound healing: A review. Wound Repair Regen 2004;12:591-9.

19. Yannas IV. Similarities and differences between induced organ regeneration in adults and early foetal regeneration. J R Soc Interface 2005;2:403-17.

20. Cornwell KG, Landsman A, James KS. Extracellular matrix biomaterials for soft tissue repair. Clin Podiatr Med Surg 2009;26:507-23. 